\title{
Impact of fibrinolytics on the outcome of empyema in South African children
}

\author{
M Zampoli, MD; A Kappos, MD; C Verwey, MD; R Mamathuba, MD; H J Zar, PhD \\ Division of Paediatric Pulmonology, Department of Paediatrics and Child Health, Faculty of Health Sciences, University of Cape Town, South Africa
}

Corresponding author: M Zampoli (m.zampoli@uct.ac.za)

\begin{abstract}
Background. Childhood pneumonia is common in all countries, and empyema is one of the commonest complications. The role of routine intrapleural fibrinolytics in the management of childhood empyema is not well established in low- and middle-income countries.

Methods. We did a prospective observational study of children sequentially hospitalised with empyema between December 2006 and December 2011 in South Africa (SA). Intrapleural tissue plasminogen activator (TPA), administered according to a standard protocol, was introduced in September 2009. Outcomes in children treated with TPA after 2009 were compared with the historical cohort not treated with TPA who met the treatment criteria.

Results. One hundred and forty-two children with empyema, median age 17 months (interquartile range 8 - 43), were admitted during the study period. Excluding children who did not have a chest tube inserted and those in whom fibrinolysis was contraindicated, there were 99 patients, 52 of whom received fibrinolytics. Clinical characteristics and empyema aetiology were similar in those who received fibrinolysis and those who did not. Eighteen children (38.3\%) not treated with TPA required surgery v. $5(9.6 \%)$ treated with TPA (relative risk 0.25; $95 \%$ confidence interval $0.1-0.6$ ). The median duration of hospitalisation was similar in both groups. Complications occurred rarely and with a similar incidence in both groups. In-hospital mortality was low, with two deaths in each group.

Conclusion. Intrapleural TPA resulted in a four-fold reduction in surgery. Fibrinolytics should be used for management of empyema in children in SA.

S Afr Med J 2015;105(7):549-553. DOI:10.7196/SAMJnew.7796
\end{abstract}

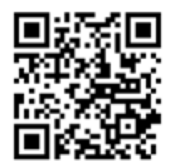

Community-acquired pneumonia (CAP) is a leading cause of childhood morbidity and mortality, with an estimated global incidence of 0.22 episodes per child year in 2010 in low-and middle-income countries (LMICs) ${ }^{[1]}$ Empyema is an important complication of CAP, with Streptococcus pneumoniae and Staphylococcus aureus leading causes. Although an increase in the incidence of empyema has been reported from some high-income countries following introduction of 7-valent pneumococcal conjugate vaccine (PCV7), the incidence of empyema in the USA following introduction of 13-valent PCV (PCV13) has dropped by $50 \%$ in children $<2$ years of age. ${ }^{[2-4]}$ In South Africa (SA), the incidence of invasive pneumococcal disease has similarly fallen following the introduction of PCV13 into the national immunisation programme in 2011. ${ }^{[5,6]}$

Intrapleural fibrinolytic therapy as first-line treatment of empyema has been reported in two randomised controlled trials in children. In a study in the UK, Thomson et al. ${ }^{[7]}$ reported that urokinase resulted in a 2-day reduction in length of hospital stay compared with normal saline, but the rate of treatment failure was similar in both treatment groups. Singh et al. ${ }^{[8]}$ compared streptokinase with normal saline and reported that the need for surgical decortication was higher at 30 days in the normal-saline groups. Intrapleural fibrinolysis as the primary intervention for empyema has an outcome equivalent to videoassisted thorascopic surgery (VATS). ${ }^{[9]}$ However, surgery, whether VATS or thoracotomy, is invasive and expensive, and requires specific expertise that is often unavailable in LMICs. ${ }^{[10]}$

There are no data on the efficacy of fibrinolytics in an African setting where the burden of childhood pneumonia is high and comorbid illness such as HIV infection, tuberculosis (TB) and malnutrition lead to more severe disease. The aim of this study was to compare the outcome of children with empyema before and after routine fibrinloysis was introduced at a tertiary paediatric hospital in SA in 2009.

\section{Methods}

\section{Study design and setting}

This was a prospective study of children sequentially hospitalised for empyema between December 2006 and December 2011 at Red Cross War Memorial Children's Hospital (RCWMCH), a large public paediatric referral hospital in Cape Town, SA. Eligible patients were identified through direct referral to the pulmonology service and daily active surveillance of all admissions for empyema. Patient care was supervised by the same medical and surgical teams throughout the study period.

Children with pleural effusion after surgery or trauma were excluded. Patients admitted directly to the paediatric intensive care unit on presentation were also excluded. Children presenting with confirmed, suspected or probable $\mathrm{TB}$ pleural effusions were not enrolled. However, those with TB presenting clinically as empyema where TB was not initially suspected but was confirmed by culture after the child received fibrinolytics were included in the treatment analysis.

\section{Empyema definition and management}

Empyema was diagnosed if the pleural fluid met any of the following criteria: frank pus or turbid fluid on inspection; neutrophil predominance or pus cells present on microscopy; bacterial organism seen by Gram stain or isolated by culture; or loculated pleural collection on chest ultrasound. TB empyema was diagnosed if the pleural effusion met any of the above criteria and Mycobacterium tuberculosis was detected in the pleural fluid by direct microscopy or liquid culture.

Routine management of empyema included general supportive measures and broad-spectrum antibiotics for 14 days (intravenous followed by oral). Empirical intravenous ampicillin $(50 \mathrm{mg} / \mathrm{kg}$ 6-hourly) and cloxacillin ( $50 \mathrm{mg} / \mathrm{kg}$ 6-hourly) was the standard of care. 
Children with suspected or proven staphylococcal empyema were treated for 6 weeks with cloxacillin or the oral equivalent. An aminoglycoside (gentamycin $5 \mathrm{mg} / \mathrm{kg} / \mathrm{d}$ ) was added in children who were malnourished, HIV-infected or under 3 months of age, according to local guidelines. Antibiotics were adjusted according to clinical response and microbiological results. TB treatment was initiated in cases where TB co-infection was clinically suspected or microbiologically proven.

Before 2009, empyema was managed by chest tube drainage without fibrinloysis. In 2009 , a protocol for intrapleural fibrinolysis was introduced. The protocol and indications for chest tube insertion, fibrinloysis and referral for surgery are summarised in Fig. 1. A chest tube to drain the pleural space was inserted under sedation and local anaesthesia if any of the following were present: large pleural effusion causing symptoms (pain or tachypnoea) or mediastinal shift; turbid fluid or pus aspirated during pleurocentesis; pleural effusion associated with clinical signs of sepsis; pyopneumothorax; or effusion with persistent pyrexia ( $>48$ hours) despite appropriate antibiotic treatment. Large hardbore chest tubes (10 - 14F gauge) attached to an underwater drainage system were initially the standard of care, but percutaneous pigtail catheters ( $12 \mathrm{~F}$ gauge) became available in 2010 and were subsequently preferentially used.

After confirmation of the correct chest tube position by X-ray and exclusion of contraindications (Fig. 1), tissue plasminogen activator (TPA) (Alteplase, Boehringer Ingelheim) was instilled into the pleura via the chest tube according to a standard protocol (Fig. 1) within 24 hours after insertion of the chest tube. A dose of $4 \mathrm{mg}$ TPA in $40 \mathrm{~mL} 0.9 \%$ saline $(2 \mathrm{mg}$ in $20 \mathrm{~mL}$ for children $<5 \mathrm{~kg}$ ) was instilled once a day for 3 consecutive days (Fig. 1).

\section{Clinical data collection, investigations and outcomes}

Clinical, aetiological and outcome data were prospectively collected. All patients had a chest X-ray done on admission. A chest ultrasound scan was requested at the discretion of the attending clinician. Pleural fluid, obtained by pleurocentesis or at the time of chest tube insertion, was sent for routine microscopy and bacterial culture. $\mathrm{TB}$ investigations, including a tuberculin skin test, gastric lavage and pleural fluid TB microscopy and culture, were performed when TB was clinically suspected and at the discretion of the attending clinicians. Analysis of the pleural fluid included total protein

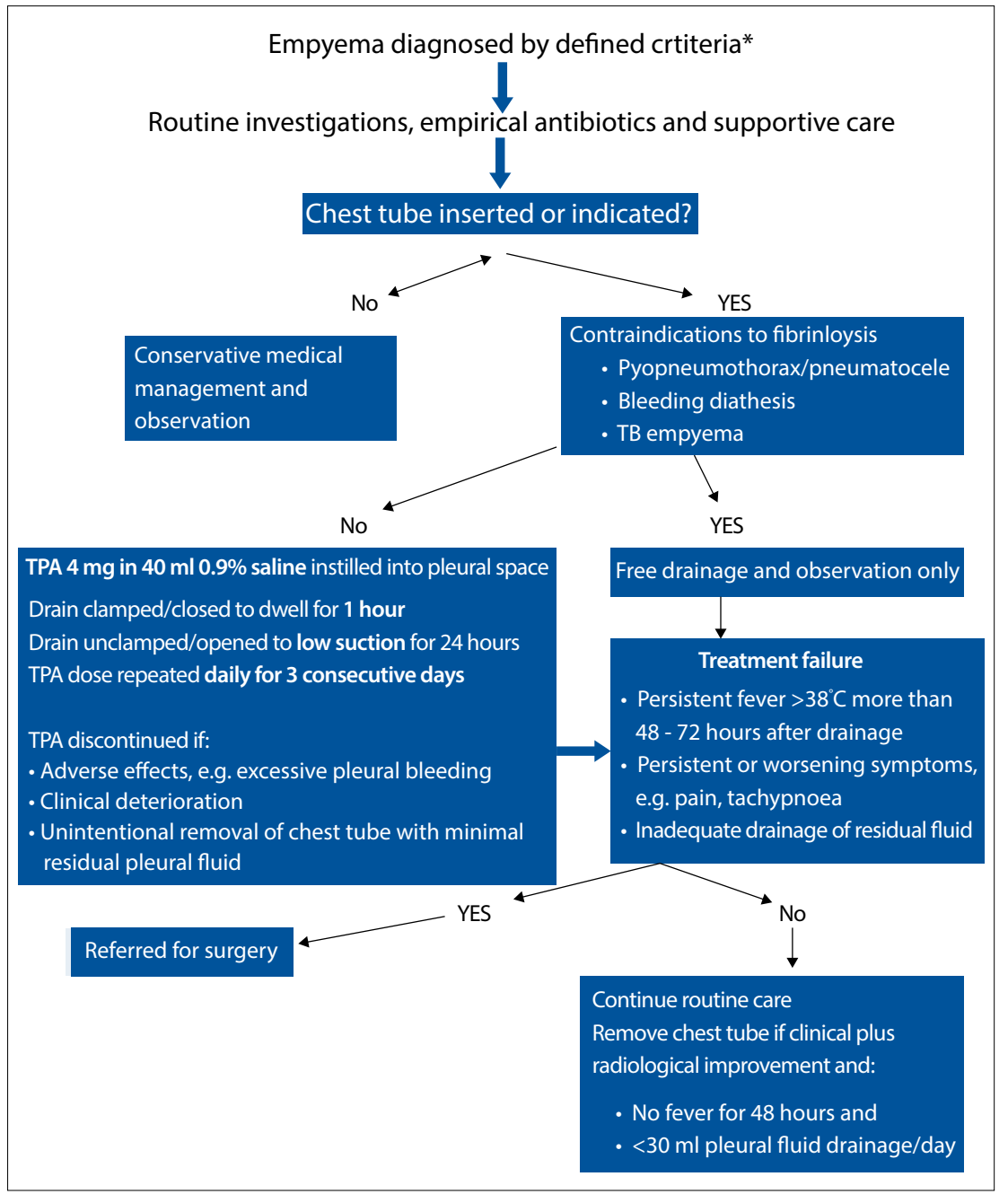

Fig. 1. Chest tube and fibrinloysis protocol. ( ${ }^{\star}$ Empyema definition: frank pus or turbid fluid; neutrophil predominance, pus cells or bacteria on microscopy; or loculated pleural collection on chest ultrasound scan.)

and lactate dehydrogenase where possible. A blood culture was performed routinely. HIV testing with consent was routinely done according to the current standard of care, unless the HIV status of the child was known. An HIV DNA polymerase chain reaction (Amplicor HIV-1 DNA test version 1.5; Roche Diagnostics, GmbH, Germany) was done in those younger than 18 months, and an HIV enzyme-linked immunosorbent assay (ArchitectHIV Ag/Ab Combo ELISA; Abbott Laboratories, USA) in older children. A CD4 count was done in HIV-infected children. Weight-for-age $z$-scores (WAZs) (World Health Organization) were calculated for children $<5$ years of age; malnutrition was defined as a $W A Z<-2$.

The primary outcome was need for surgical intervention. The indications for surgical referral are described in Fig. 1. Secondary outcomes were length of hospital stay and in-hospital mortality. Complications relating to the underlying empyema and treatmentrelated complications were also recorded.
The study was approved by the Human Research Ethics Committee of the Faculty of Health Sciences, University of Cape Town (HREC 230/2009).

\section{Statistical analysis}

Data were analysed using STATA (version 10.0, Statcorp, USA). Continuous variables were expressed as means (standard deviation (SD)) for normally distributed variables or medians and interquartile ranges (IQRs) for non-normally distributed variables. The Mann-Whitney $U$-test was used for comparing non-normally distributed measures. Categorical variables were compared using the $\chi^{2}$ test. Relative risk (RR) and 95\% confidence intervals (CIs) were calculated where applicable.

\section{Results \\ Clinical characteristics}

One hundred and forty-two children (59 in the pre-fibrinolysis period) with a median age of 17.4 months (IQR 8 - 43) were hospitalised 
for empyema during the study period; 81 $(57.0 \%)$ were male. Most $(n=112,78.9 \%)$ were referred from a primary care setting and $30(21.1 \%)$ from other hospitals. Sixtysix/127 (52.0\%) received oral, intramuscular or intravenous antibiotics prior to admission to RCWMCH. PCV immunisation status could not be determined in 11 cases; 6 children were partially immunised and the remainder were unimmunised. Twenty-eight patients (19.7\%) were HIV-infected, with a mean CD4 count of $18.4 \%$ (SD 9.5\%), and 44/114 children (38.6\%) were malnourished. Pyopneumothorax was present in 22 cases $(15.5 \%)$ and pneumatocele(s) in $9(6.3 \%)$.

\section{Empyema aetiology}

The culture-positive rates of pleural fluid and blood culture were 45/142 (31.7\%) and $33 / 132$ (25.0\%), respectively (Table 1 ). The commonest bacterial pathogens identified on blood or pleural culture were $S$. aureus and S. pneumoniae (Table 1). Ninety and 104 patients, respectively, had gastric lavage and pleural fluid investigated for TB. Overall, 12/142 (8.4\%) children had culture-confirmed $\mathrm{TB}$, of whom 10/104 (9.6\%) were diagnosed with TB empyema. Two additional cases of culture-confirmed TB were detected through gastric lavage cultures. Bacterial-TB empyema was confirmed by culture in 5 patients.

\section{Clinical course and outcome}

One hundred and thirty-five patients (95.1\%) had a chest tube inserted and 9 (6.6\%) required two or more chest tubes during their hospitalisation. The median duration of chest tube placement was 5 days (IQR 3 - 10). Large-bore hard chest tubes were used in most patients $(107,79.3 \%)$ and pigtail catheters in 27 (20.0\%), all in the TPA treatment group. Nineteen patients (13.4\%) were referred to the intensive care unit. Overall, 29 (20.5\%) patients needed surgery and 6 died in hospital (4.2\%).

Excluding patients who did not have a chest tube inserted because their disease was mild and those in whom fibrinolysis was contraindicated (22 pyopneumothorax, 9 pneumatoceles and 5 TB empyema), data on 99 patients from the pre- and postfibrinolysis periods (TPA 52, no TPA 47) were available for comparative analysis (Fig. 2). Baseline clinical characteristics and empyema aetiology were similar in the two groups (Table 2). Eighteen of 47 children (38.3\%) not treated with fibrinolytics required surgery compared with 5/52 (9.6\%) of those treated with TPA (RR 0.25; 95\% CI $0.1-0.6)$. There was a trend towards shorter hospital stay in the TPA group, but the difference was not statistically significant

Table 1. Bacterial isolates from pleural fluid and blood culture ( $N=142$ patients)

\begin{tabular}{llll}
\hline Pleural fluid culture & $\begin{array}{l}\text { Frequency } \\
\boldsymbol{n}(\%)\end{array}$ & Blood culture & $\begin{array}{l}\text { Frequency } \\
\boldsymbol{n}(\%)\end{array}$ \\
\hline Staphylococcus aureus & $19(13.4)$ & S. pneumoniae & $18(12.7)$ \\
Streptococcus pneumoniae & $14(9.9)$ & S. aureus & $9(6.3)$ \\
Other streptococci & $3(2.1)$ & H. influenzae & $2(1.4)$ \\
Staphylococcus aureus/Escherichia coli & $1(0.7)$ & S. aureus/K. pneumoniae & $1(0.7)$ \\
Haemophilus influenzae & $1(0.7)$ & S. pneumoniae/S. aureus & $1(0.7)$ \\
H. influenzae/E. coli & $1(0.7)$ & S. hominis & $1(0.7)$ \\
Klebsiella pneumoniae & $1(0.7)$ & Pantoea spp. & $1(0.7)$ \\
Anaerobes & $1(0.7)$ & S. pyogenes & $1(0.7)$ \\
No growth & $94(66.2)$ & No growth & $98(69.0)$ \\
Not done & $7(4.9)$ & Not done & $10(7.0)$
\end{tabular}

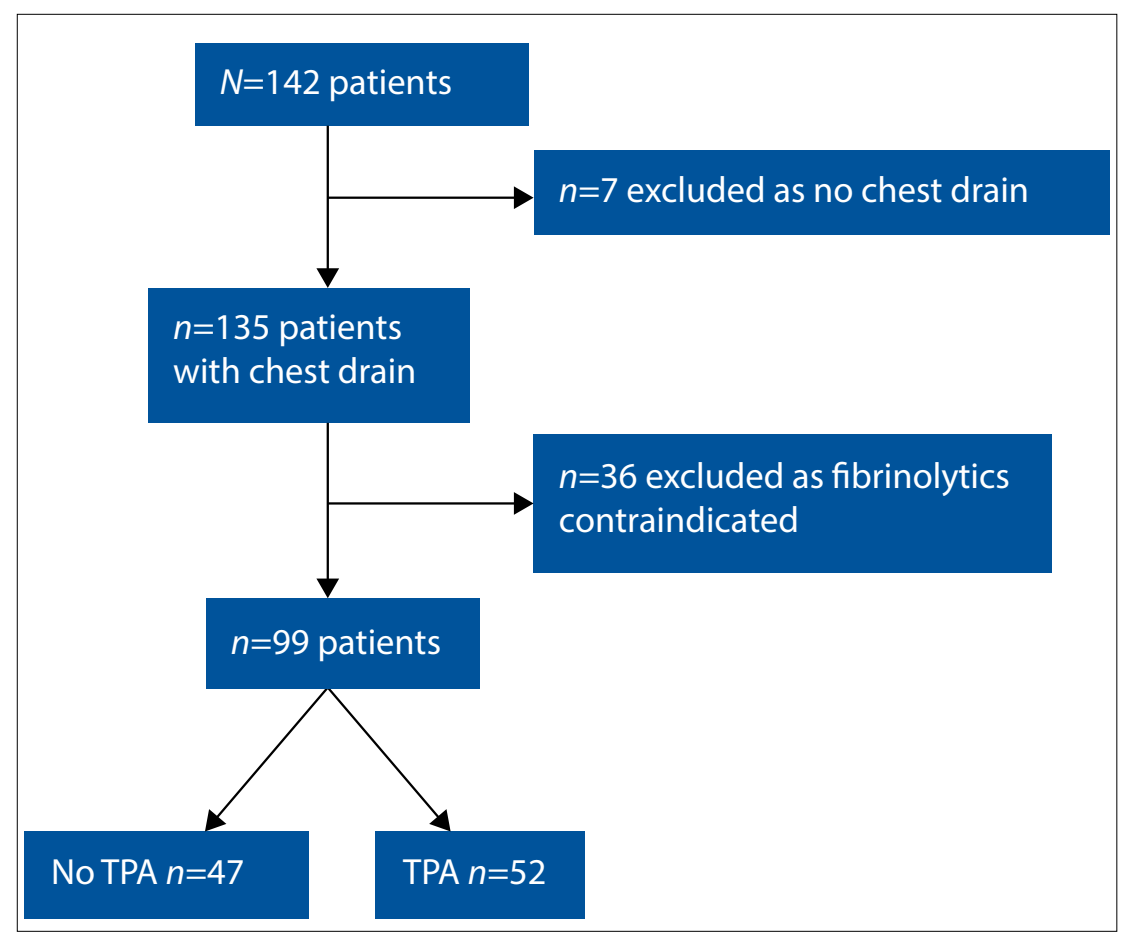

Fig. 2. Study population selection.

(Table 3). Surgery, however, was associated with a longer hospital stay (median 16 days (IQR 13 - 29) v. 12 days (IQR 8 - 17); $p=0.001)$. The median time to surgery from admission was similar in the two groups (7 days each). Complications relating to empyema or treatment occurred rarely in both groups (Table 3 ).

Compared with children who were eligible for fibrinolysis, a longer period of hospitalisation (median 18 days (IQR 11 29) v. 12 days (IQR 8 - 16); $p<0.01$ ), more disease complications (10/32 (31.3\%) v. $11 / 102(10.8 \%) ; p<0.01)$ and a longer time to surgery (median 13 days (IQR 9 20) v. 7 days (IQR $4-8$ ); $p=0.02$ ) were observed in children in whom fibrinolysis was contraindicated. Six of these 36 patients (16.7\%) needed surgery. Mortality was low and similar to the rest of the cohort $(2 / 36$ (5.6\%) v. $4 / 106$ (3.8\%); $p=0.6)$.

\section{Discussion}

This study found a four-fold reduction in surgical intervention with the implementation of routine fibrinloysis using TPA in SA children with empyema. The results also suggest a trend towards shorter hospital stay and fewer chest drains in children receiving fibrinolytics. This is the first study describing the use of fibrinolytics in an African setting where the prevalence of childhood malnutrition, HIV and infectious diseases is high, as shown by the comorbidity 
Table 2. Baseline and clinical characteristics of patients with and without TPA treatment

\begin{tabular}{|c|c|c|c|}
\hline & $\begin{array}{l}\text { TPA } \\
(N=52)\end{array}$ & $\begin{array}{l}\text { No TPA } \\
(N=47)\end{array}$ & $p$-value \\
\hline Age (months), median (IQR) & $21(8-42)$ & $17(8-52)$ & 0.9 \\
\hline HIV infected, $n(\%)$ & $14 / 50(28.0)$ & $10 / 40(25.0)$ & 0.7 \\
\hline WAZ ( $<5$ years), median (IQR) & $-1.08(-2.4--0.3)$ & $-1.72(-2.6--0.4)$ & 0.3 \\
\hline Community referral, $n(\%)$ & $42(80.8)$ & $37(78.7)$ & 0.8 \\
\hline Prior antibiotics, $n(\%)$ & $24 / 52(46.2)$ & $24 / 40(60.0)$ & 0.1 \\
\hline No PCV, $n(\%)$ & $43 / 47(91.5)$ & $46 / 46(100.0)$ & 0.1 \\
\hline Streptococcus pneumoniae ${ }^{\star}, n(\%)$ & $11 / 52(21.2)$ & $10 / 47(21.3)$ & 0.9 \\
\hline Staphylococcus aureus,$n(\%)$ & $7 / 52(13.5)$ & $5 / 47(10.6)$ & 0.6 \\
\hline TB co-infection, $n(\%)$ & $3 / 30(10.0)$ & $2 / 35(5.7)$ & 0.6 \\
\hline Total WCC $\left(\times 10^{9} / \mathrm{L}\right)$, median $(\mathrm{IQR})$ & $18.5(11.5-31.2)$ & $24.4(16.9-35.4)$ & 0.07 \\
\hline $\begin{array}{l}\text { Haemoglobin }(\mathrm{g} / \mathrm{dL}) \text {, median } \\
\text { (IQR) }\end{array}$ & $8.7(7.2-10)(n=49)$ & $\begin{array}{l}9(8-10) \\
(n=46)\end{array}$ & 0.4 \\
\hline $\begin{array}{l}\text { C-reactive protein }(\mathrm{mg} / \mathrm{L}) \text {, mean } \\
(\mathrm{SD})\end{array}$ & $\begin{array}{l}258(101) \\
(n=26)\end{array}$ & $\begin{array}{l}193(91) \\
(n=22)\end{array}$ & 0.02 \\
\hline $\begin{array}{l}\text { Fluid lactate dehydrogenase (U/L), } \\
\text { median (IQR) }\end{array}$ & $\begin{array}{l}1251(596-5040) \\
(n=39)\end{array}$ & $\begin{array}{l}2743\left(\begin{array}{llll}1 & 181 & -10 & 144) \\
(n=14)\end{array}\right.\end{array}$ & 0.2 \\
\hline $\begin{array}{l}\text { Fluid total protein }(\mathrm{g} / \mathrm{L}) \text {, mean } \\
(\mathrm{SD})\end{array}$ & $\begin{array}{l}46(17) \\
(n=36)\end{array}$ & $\begin{array}{l}49(19.5) \\
(n=14)\end{array}$ & 0.5 \\
\hline Underlying comorbidity, $n(\%)$ & $7 / 52(13.5)$ & $6 / 47(12.8)$ & 0.9 \\
\hline
\end{tabular}

Table 3. Outcomes of patients treated with and without fibrinolysis

\begin{tabular}{|c|c|c|c|}
\hline & $\begin{array}{l}\text { TPA } \\
(N=52)\end{array}$ & $\begin{array}{l}\text { No TPA } \\
(N=47)\end{array}$ & $\begin{array}{l}\text { Statistical } \\
\text { significance }\end{array}$ \\
\hline Surgery, $n(\%)$ & $5(9.6)$ & $18(38.3)$ & $\begin{array}{l}\mathrm{RR}(95 \% \mathrm{CI}) \\
0.25(0.1-0.6)\end{array}$ \\
\hline Days in hospital, median (IQR) & $9.5(7-16)$ & $12(10-20)$ & $p=0.08$ \\
\hline Disease complications, $n(\%)$ & $5(9.6)$ & $5(10.6)$ & NS \\
\hline Treatment complications, $n(\%)$ & $4(7.7)^{*}$ & $2(4.3)^{\dagger}$ & NS \\
\hline Mortality, $n(\%)$ & $2(3.8)$ & $2(4.2)$ & NS \\
\hline
\end{tabular}

present in children. The prevalence of TB was nearly $10 \%$, highlighting the importance of $\mathrm{TB}$ as an unrecognised cause of empyema in this setting.

These findings are consistent with other reports, mostly from high-income countries, despite the high rate of comorbid illness, including HIV and malnutrition, in children in our study. Several observational studies have compared the outcome of empyema in children treated with fibrinolytics (TPA, urokinase or streptokinase) with historical cohorts not treated with fibrinolytics. With the exception of an Australian study, all reported a similar reduction in need for surgical intervention. ${ }^{[11-14]}$
The pre-fibrinolysis treatment failure rate reported in our study (38.3\%) is higher than the average rate $(23.6 \%)$ found in a large meta-analysis of non-operative outcomes of 2793 empyema cases published between 1982 and 2004. ${ }^{[15]}$ Mortality and complication rates were $3.3 \%$ and $5.6 \%$, respectively, similar to our study. The rate of treatment failure without fibrinloysis is variable, however, and has been reported in more recent studies to be as high as $74-87 \% .^{[13,16]}$ The higher than average treatment failure rate in the pre-fibrinolytics era in our study could be attributed to several factors. First, children in our cohort were considerably younger than those in most published studies (median age 17.4 months v. 5 years). ${ }^{[15]}$ Furthermore, the high prevalence of HIV infection and malnutrition in our cohort are probable contributing factors. Complicated or severe empyema is a consequence of the increased risk of severe invasive pneumococcal disease in children who are young, HIVinfected or malnourished. ${ }^{[17]}$ Higher rates of empyema in indigenous children compared with national populations have been reported elsewhere, suggesting that poverty, environmental exposure or malnutrition contribute to the pathogenesis and severity of empyema. ${ }^{[18]}$ Secondly, referral for surgery in our practice was relatively early (median 7 days), increasing the likelihood of treatment failure as defined by our protocol. Lastly, $21.1 \%$ of patients were referred from other hospitals and represented complicated empyema cases. However, direct comparison of treatment failure rates between studies cannot be made, as definitions of treatment failure and indications for surgery differ between studies.

The fibrinloysis treatment failure rate of $9.6 \%$ in our study is comparable to other published reports. However, few prospective paediatric studies define failure of primary fibrinolysis as the primary outcome. Need for surgery is an important outcome in children, especially in settings such as subSaharan Africa, where paediatric surgical resources are scarce. Two studies comparing fibrinolysis with VATS reported a fibrinolysis failure rate of $17 \%$ each. ${ }^{[19,20]}$ Other prospective studies comparing fibrinolysis with normal saline or various doses of urokinase report fibrinolysis failure rates of 7 - 24\%. ${ }^{[7,8,21]}$ A meta-analysis published in 2005 calculated the average failure rate of 64 empyema cases treated with primary fibrinolysis to be $9.3 \%$. ${ }^{[15]}$

\section{Study limitations}

The main limitation of this study is the use of a historical cohort as the control group. However, the prospective approach to patient recruitment, data collection and implementation of a standard fibrinolysis protocol strengthens the validity of our findings. Furthermore, the study was conducted at a single centre where senior medical staff and treatment protocols remained unchanged throughout the study period. The introduction of PCV7 in the national immunisation programme in 2009 may have influenced the spectrum of empyema in our cohort. However, this is unlikely to have had an impact on the study findings, as very few patients had received 
at least one dose of PCV7 because most were not an age eligible for immunisation when this was introduced into the programme and there was no catch-up campaign during this period. A second limitation is the potential bias towards delayed surgery and longer hospitalisation in the treatment group, but the similar time to surgery in both arms excludes this bias in our study. Lastly, although we attempted to exclude suspected or known TB empyema cases from treatment and analysis, a few children with TB empyema inadvertently received fibrinolysis because the diagnosis was not suspected at the time of presentation. These few cases were included in the analysis, as all were co-infected with a bacterial pathogen.

\section{Conclusion}

The findings of our study have important implications for managing childhood empyema in settings where access to paediatric surgical expertise is limited. This study adds to the accumulating evidence for a beneficial role of primary fibrinolysis in childhood empyema. Fibrinolytics should be used for management of children with empyema who require drainage in LMIC settings.

Acknowledgements. We thank Henri Carrara for his expert guidance and review of the statistical methodology in this study, and the staff of RCWMCH for their support of the study.

Funding information. None/not applicable.

Prior abstract presentation. This paper was presented as a poster at the Congress International of Paediatric Pulmonology (CIPP), 26 - 30 June 2014, Bruges, Belgium. Abstract published in Pediatric Pulmonology 2014;49(S37):S66.

\footnotetext{
References

1. Rudan I, O'Brien KL, Nair H, et al. Epidemiology and etiology of childhood pneumonia in 2010 Estimates of incidence, severe morbidity, mortality, underlying risk factors and causative pathogens for 192 countries. J Glob Health 2013;3(1):10401. [http://dx.doi.org/10.7189/jogh.03.010401; 10.7189/ jogh.03.010401]

2. Li ST, Tancredi DJ. Empyema hospitalizations increased in US children despite pneumococcal conjugate vaccine. Pediatrics 2010;125(1):26-33. [http://dx.doi.org/10.1542/peds.2009-0184]
}

3. Fletcher MA, Schmitt HJ, Syrochkina M, Sylvester G. Pneumococcal empyema and complicated pneumonias: Global trends in incidence, prevalence, and serotype epidemiology. Eur J Clin Microbiol Infect Dis 2014;33(6):879-910. [http:///x.doi.org/10.1007/s10096-014-2062-6]

4. Simonsen L, Taylor RJ, Schuck-Paim C, Lustig R, Haber M, Klugman KP. Effect of 13-valent pneumococcal conjugate vaccine on admissions to hospital 2 years after its introduction in the USA: A time series conjugate vaccine on admissions to hospital 2 years after its introduction in the USA: A time seties
analysis. Lancet Respir Med 2014;2(5):387-394. [http://dx.doi.org/10.1016/S2213-2600(14)70032-3]

analysis. Lancet Respir Med 2014;2(5):387-394. [http://dx.doi.org/10.1016/S2213-2600(14)70032-3]
5. Von Gottberg A, de Gouveia L, Tempia S, et al. Effects of vaccination on invasive pneumococcal disease 5. Von Gottberg A, de Gouveia L, Tempia S, et al. Effects of vaccination on invasive pneumococcal disease
in South Africa. N Engl J Med 2014; 371(20):1889-1899. [http://dx.doi.org/10.1056/NEJMoa1401914] 6. Madhi SA, Bamford L, Ngcobo N. Effectiveness of pneumococcal conjugate vaccine and rotavirus vaccine introduction into the South African public immunisation programme. S Afr Med J 2014;104(3 Suppl 1):228-234. [http://dx.doi.org/10.7196/SAMJ.7597]

7. Thomson AH, Hull J, Kumar MR, Wallis C, Balfour Lynn IM. Randomised trial of intrapleural urokinase in the treatment of childhood empyema. Thorax 2002;57(4):343-347. [http://dx.doi. org/10.1136/thorax.57.4.343]

8. Singh M, Mathew JL, Chandra S, Katariya S, Kumar L. Randomized controlled trial of intrapleural streptokinase in empyema thoracis in children. Acta Paediatr 2004;93(11):1443-1445. [http://dx.doi. org/10.1080/08035250410033989]

9. Mahant S, Cohen E, Weinstein M, Wadhwa A. Video-assisted thorascopic surgery vs chest drain with fibrinolytics for the treatment of pleural empyema in children: A systematic review of randomized controlled trials. Arch Pediatr Adolesc Med 2010;164(2):201-203. [http://dx.doi.org/10.1001/
a controlled trials. Arch

10. Cohen E, Weinstein M, Fisman DN. Cost-effectiveness of competing strategies for the treatment of pediatric empyema. Pediatrics 2008;121(5):e1250-e1257. [http://dx.doi.org/10.1542/peds.2007-1886]

11. Yao CT, Wu JM, Liu CC, Wu MH, Chuang HY, Wang JN. Treatment of complicated parapneumonic pleural effusion with intrapleural streptokinase in children. Chest 2004;125(2):566-571. [http://dx.doi. org/10.1378/chest.125.2.566]

12. Van Loo A, van Loo E, Selvadurai H, Cooper P, van Asperen P, Fitzgerald DA. Intrapleural urokinase versus surgical management of childhood empyema. J Paediatr Child Health 2014;50(10):823-826. [http://dx.doi.org/10.1111/j.1440-1754.2010.01931.x]

13. Faber DL, Best LA, Orlovsky M, Lapidot M, Nir RR, Kremer R. Streptokinase fibrinolysis protocol: The advantages of a non-operative treatment for stage II pediatric empyema patients. Isr Med Assoc J 2012;14(3):157-161.

14. Gasior AC, Knott EM, Sharp SW, Ostlie DJ, Holcomb GW 3rd, St Peter SD. Experience with an evidence-based protocol using fibrinolysis as first line treatment for empyema in children. J Pediatr evidence-based protocol using fibrinolysis as first line treatment for empyem
Surg 2013;48(6):1312-1315. [http://dx.doi.org/10.1016/j.jpedsurg.2013.03.029]

15. Avansino JR, Goldman B, Sawin RS, Flum DR. Primary operative versus nonoperative therapy for pediatric 5. Avansino JR, Goldman B, Sawin RS, Flum DR. Primary operative versus nonoperative therapy for pediatric
empyema: A meta-analysis. Pediatrics 2005;115(6):1652-1659. [http://dx.doi.org/10.1542/peds.2004-1405] 16. Tagbo O, Uchenna O, Anthony H. Childhood parapneumonic pleural effusion in Enugu. Niger Postgrad Med J 2005;12(1):28-32

17. Von Gottberg A, Cohen C, de Gouveia L, et al. Epidemiology of invasive pneumococcal disease in the pre-conjugate vaccine era: South Africa, 2003-2008. Vaccine 2013;31(38):4200-4208. [http://dx.doi. org/10.1016/j.vaccine.2013.04.077]

18. Singleton RJ, Holman RC, Wenger J, et al. Trends in hospitalization for empyema in Alaska native children younger than 10 years of age. Pediatr Infect Dis J 2011;30(6):528-530. [http://dx.doi. org/10.1097/INF.0b013e3182075e74

19. Sonnappa S, Cohen G, Owens CM, et al. Comparison of urokinase and video-assisted thoracoscopic surgery for treatment of childhood empyema. Am J Respir Crit Care Med 2006;174(2):221-227. [http:// dx.doi.org/10.1164/rccm.200601-027OC]

20. St Peter SD, Tsao K, Spilde TL, et al. Thoracoscopic decortication vs tube thoracostomy with fibrinolysis for empyema in children: A prospective, randomized trial. J Pediatr Surg 2009;44(1):106-111. [http:// dx.doi.org/10.1016/j.jpedsurg.2008.10.018]

21. Wang IN, Yao CT, Yeh CN, et al. Once-daily vs. twice-daily intrapleural urokinase treatment of complicated parapneumonic effusion in paediatric patients: A randomised, prospective study. Int $J$ Clin Pract 2006;60(10):1225-1230. [http://dx.doi.org/10.1111/j.1742-1241.2006.01110.x]

Accepted 27 January 2015. 